\title{
Effect of three different jaw positions on postural stability during standing
}

\author{
Ahmad H. Alghadir, PT, PhD \\ Hamayun Zafar, PT, PhD ${ }^{\mathrm{a}, \mathrm{b}}$ \\ Zaheen A. Iqbal, MPT
}

\begin{abstract}
${ }^{a}$ Rehabilitation Research Chair, Department of Rehabilitation Sciences, College of Applied Medical Sciences, King Saud University, Riyadh, Saudi Arabia

${ }^{b}$ Department of Odontology, Clinical Oral Physiology, Umea University, Umea, Sweden
\end{abstract}

Correspondence to: Zaheen A. Iqbal

E-mail: z_iqbal001@yahoo.com

\section{Summary}

Studies in the literature show that jaw and neck regions are linked anatomically, biomechanically and neurologically. Voluntary clenching has been shown to improve muscle strength and performance of various motor tasks. Information from the neck sensory-motor system is reported to be important for posture. Hence it is reasonable to believe that activation of the jaw sensorymotor system has the potential to modulate posture. In a sample of 116 healthy subjects, we compared center of gravity (COG) velocity during quiet standing on a foam surface during three test positions: i) resting jaw, ii) open jaw, and iii) clenching; these were tested in two conditions: with eyes open and with eyes closed. The COG velocity decreased significantly during clenching in comparison to both open and resting jaw positions $(p<0.0001)$. This suggests that the jaw sensory-motor system can modulate postural mechanisms. We conclude that jaw clenching can enhance postural stability during standing on an unstable surface in both the presence and absence of visual input in healthy adults and suggest that this should be taken into consideration in treatment and rehabilitation planning for patients with postural instability.

KEY WORDS: balance, center of gravity (COG) velocity, clenching, jaw position, postural stability

\section{Introduction}

Body posture has been shown to be affected by various factors, including vision, trigeminal afferents, head-neck position and proprioception (Bolmont et al., 2002; Kantor et al., 2001; Kogler et al., 2000; Wada et al., 2001; Bracco et al., 1998; Gangloff et al., 2000; Gangloff and Perrin, 2002; Milani et al., 2000; Colledge et al., 1994). Information from the sensorymotor system of the neck is reported to be important for maintaining postural stability (Abrahams, 1977). Patients who have sustained a neck trauma, e.g. those with whiplash-associated disorders, can present, along with other symptoms, gait disturbances, dizziness and balance impairments (Abrahams, 1977; Kogler et al., 2000). These findings indicate an important role of the neck sensory-motor system in the control of body posture and balance.

Purposeful and task-related movements of the body segments require coordinated actions of various joints, muscles, nerves and receptors (Zafar et al., 2002). The muscles of the jaw and the neck-shoulder complex have been observed to co-contract during mandibular movements and clenching (Davies, 1979; Clark et al., 1993; Widmalm et al., 1988). This integrated activation of jaw and neck muscles is suggested to be controlled by a common central nervous network (Zafar et al., 2000; Eriksson et al., 1998). Other studies have highlighted the anatomical, biomechanical and neurological link between the jaw and neck regions (Brodie, 1950; Chang et al., 1988). Voluntary teeth clenching has been shown to improve the performance of various motor tasks (e.g. to enhance sports performance) and to increase muscle strength (e.g. hand grip) (Ebben, 2006; Hiroshi, 2003; Cherry et al., 2010). It has also been reported that dental occlusion status contributes to the maintenance of postural balance (Milani et al., 2000; Hosoda et al., 2007). Previous studies allow us to infer that the jaw and neck systems are functionally related. Hence it is reasonable to believe that activation of the jaw sensory-motor system has the potential to modulate the postural control system through the neck, directly or indirectly.

The aim of this paper was to study the effect of different jaw positions on standing postural balance in healthy adults. We hypothesize that activation of the jaw sensory-motor system with the jaw in different positions can affect postural stability during quiet standing. We studied the effect of resting, open and clenched jaw positions on center of gravity (COG) velocity during quiet standing on a foam surface with eyes open and closed. 


\section{Materials and methods}

\section{Subjects}

One hundred and sixteen healthy male subjects (average age 31.56 , SD 8.51 years; height 170.86 , SD 7.26 $\mathrm{cm}$ ) participated in the study. To be included in the study, subjects had to have complete Angle Class I or Class II dentition, and those with any signs or symptoms of balance impairment, temporomandibular joint disorders or skeletal anomalies were excluded. All the subjects were informed about the aims and procedures of study and written consent was obtained in accordance with the Declaration of Helsinki. The study fully complied with the ethical standards for human research of the King Saud University.

\section{COG velocity assessment}

The COG velocity of natural sway during standing was assessed using the NeuroCom ${ }^{\circledR}$ Balance Master (version 8.5.0, NeuroCom International Inc., Clackamas, OR, USA). This equipment has previously been used in many studies (Liston and Brouwer, 1996; Newstead et al., 2005; Chien Hu et al., 2007). The subject stands on a $46 \times 152$ $\mathrm{cm}$ force platform, and the data generated by the load cells in the force platform are collected by the interfaced computer using dedicated software. Balance Master was automatically calibrated before each testing session.

Each subject was asked to stand as still as possible on a soft, relatively unstable surface, i.e. a $50 \times 50 \times 15$ $\mathrm{cm}$ foam block placed on the force platform, with their feet comfortably together and arms by their sides. This foam block is a standard accessory of Balance Master, and has previously been used in many studies to make standing balance more challenging (Liston and Brouwer, 1996; Chien et al., 2007). For each subject, the COG velocity (expressed as deg/s) was measured during three different jaw positions: i) resting jaw, i.e., the jaw position naturally assumed when no instructions are given, ii) open jaw, i.e., with the teeth of both jaws slightly apart, and iii) clenched jaw, i.e., with the teeth of both jaws tightly closed. Data for each test position were obtained in the eyes open condition and the eyes closed condition.

The duration of the test run for each of the three positions was 10 seconds. The study procedures were explained to the subjects before any measurements were carried out. Each test position was repeated three times in each subject, and a rest of about 60 seconds was allowed between the trials. With the subject trying to stand as still as possible, the COG velocity of the natural sway was sampled at a frequency of $100 \mathrm{~Hz}$ and the mean of the three trials was used for data analysis.

\section{Data analysis}

Data were analyzed using Graph-Pad Instat 3.0 (GraphPad Software Inc., CA, USA). Means and SDs were used for descriptive statistics. The hypothesis of no difference in COG velocity during the resting jaw position, open jaw position and clenched jaw position was tested by the Friedman test. The null hypothesis was rejected at the 0.05 level of significance.

\section{Results \\ COG velocity during three test positions with eyes open and eyes closed}

The mean COG velocity during quiet standing on a relatively unstable surface varied between the three test positions, with values of 0.81 (SD 0.68), 0.74 (0.84) and $0.68(0.64)$ found for the resting jaw, open jaw and clenched jaw test positions, respectively, with eyes open. With eyes closed, mean COG velocity values of 1.58 (SD 0.64), $1.36(0.74)$ and 1.18 (0.57) were found for the resting jaw, open jaw and clenched jaw test positions, respectively (Table I).

\section{Comparison of COG velocities recorded in the three test positions}

In the eyes closed condition, the mean COG velocity showed a significant reduction in the open jaw compared with the resting jaw position $(p<0.0001)$, in the clenched jaw compared with the resting jaw position $(p<0.0001)$, and in the clenched jaw compared with the open jaw position $(p<0.0001)$ (Fig. 1).

Table I - Center of gravity velocity in 116 healthy adult males during quiet standing with eyes open (EO) and closed (EC).

\begin{tabular}{lllllll}
\hline & \multicolumn{2}{l}{ Resting jaw } & \multicolumn{2}{l}{ Open jaw } & \multicolumn{2}{c}{ Clenched jaw } \\
& EO & EC & EO & EC & EO & EC \\
\hline Mean & 0.812 & 1.588 & 0.740 & 1.386 & 0.686 & 1.189 \\
SD & 0.686 & 0.643 & 0.846 & 0.746 & 0.646 & 0.578 \\
\hline
\end{tabular}

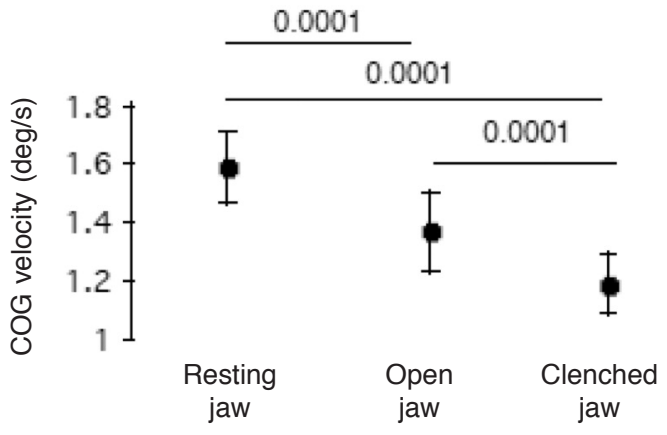

Figure 1 - Mean and $95 \%$ confidence intervals of COG velocity values of all subjects $(n=116)$ for quiet standing on a relatively unstable surface with eyes closed during three test positions. Note significant differences $(p=0.0001)$ in COG values between the three test conditions and the lowest mean value for the clenched jaw condition. 
In the eyes open condition, significant reductions in mean COG velocity were found in the open jaw compared with the resting jaw position $(\mathrm{p}<0.0001)$, and in the clenched jaw compared with the resting jaw position $(\mathrm{p}<0.0001)$ (Fig. 2).

In the eyes open condition, COG velocity decreased by $9 \%$ during the open jaw and by $16 \%$ during the clenched jaw positions, while in the eyes closed condition, it decreased by $14 \%$ during the open jaw and by $25 \%$ during the clenched jaw positions as compared to the values recorded in the resting jaw position, respectively (Fig. 3).

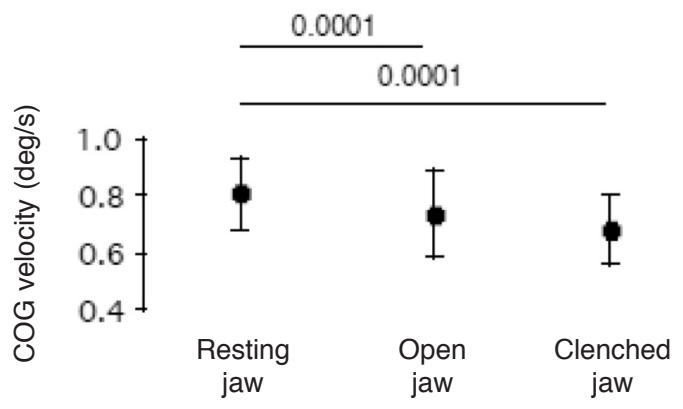

Figure 2 - Mean and 95\% confidence intervals of COG velocity values of all subjects $(n=116)$ for quiet standing on relatively unstable surface with eyes open during three test positions. Note significant differences $(p=0.0001)$ in COG values between the three test conditions and the lowest mean value for the clenched jaw condition.

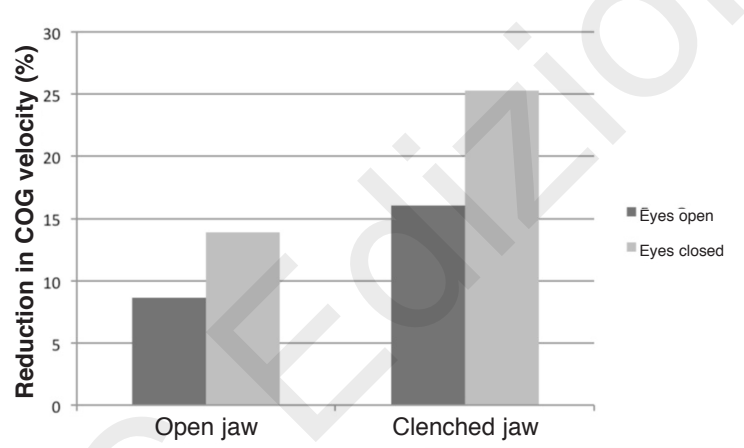

Figure 3 - In comparison to the resting jaw position, the relative reduction in COG velocity during the open jaw and clenched jaw positions with eyes open and eyes closed.

\section{Discussion}

We studied the possible effect of different jaw positions on postural balance in healthy adults standing on a relatively unstable foam surface. The results showed that the COG velocity of postural sway during standing decreased significantly during the open and clenched jaw positions compared to the resting jaw position, both with eyes open and with eyes closed.

In order to maintain postural stability, the body's balance system requires information about the body's position and motion in space (Perinetti, 2006). This information, in the form of various sensory inputs, is provided via various channels, e.g. as cutaneous information from the sole of the foot (Kavounoudias et al., 1998; Meyer et al., 2004); as visual information (Collins and De Luca, 1995), or via vestibular, ocular (Horak and Hlavacka, 2001; Wardman et al., 2003) somatosensory channels, etc. (Fransson et al., 2000, 2003; Rogers et al., 2001; Massion, 1994). There is evidence that the sensory-motor system of the jaw can influence the vestibular (Park et al., 2014), neck (Davies, 1979; Ehrlich et al., 1999) and ocular systems and can therefore also affect the postural control system (Hellmann et al., 2011). Our results, showing significant decreases in COG velocity between different jaw positions, seem to support this and show that activation of the jaw sensory-motor system can affect postural control mechanisms.

The role of visual input (Redfern et al., 2001; Edwards, 1946) and of standing surface (Mohapatra et al., 2014) in postural balance is well documented. It has been shown that standing on a surface such as foam presents a significant challenge to the postural control system as it alters the inputs to both joint receptors and cutaneous mechanoreceptors in the sole of the foot (Mohapatra et al., 2014; Blackburn et al., 2003). It is interesting to note that the subjects in our study, even when deprived of visual input and put in the more challenging situation of standing on a relatively unstable foam surface, showed decreases in COG velocity with changes in jaw position, indicating that the jaw sensory-motor system is capable of modulating the postural control system in order to stabilize the body. In comparison to what was observed in the open eyes condition, a relatively higher reduction of COG velocity during the open and clenched jaw positions as compared to the resting jaw position was observed in the eyes closed condition. This finding suggests that the sensory-motor system of the jaw can play an important role in the absence of visual input.

It is possible that occlusal changes are involved in the decline of balance ability in the elderly. Indeed, in the absence of proper occlusion, the ability of the body to adjust its COG becomes impaired (Hosoda et al., 2007). It has been reported that wearing an occlusal splint may allow patients to alter their postural attitude (Milani et al., 2000) and occlusal therapy has been shown to induce a re-equilibrium of masticatory muscles via a descending action resulting in an improved body posture (Bracco et al., 2004). According to a study on relations between dental occlusion and body balance, the myocentric jaw position (right-left muscular equilibrium) improved balance in the frontal plane compared with the centric (most closed, static) position and rest (habitual) position (Bracco et al., 2004). Teeth clenching facilitates $\mathrm{H}$ reflexes of the soleus and pretibial muscles (Miyahara et al., 1996) and a positive relationship has been reported between the extent of facilitation of the reflex and the magnitude of electromyographic activity of the masseter muscle (Takada et al., 2000). Clenching exerts a nonreciprocal tonic facilitation of the $\mathrm{H}$ reflex of the ankle extensor and flexor muscles (Fujino et al., 2010). Isometric activation of the jaw motor system, in conditions like 
maximum bite and unilateral sub maximum bite and chewing, has also been shown to affect body sway (Hellmann et al., 2011). The clenched jaw position, exerting a similar action, may also decrease the COG velocity and improve the balance of the individual through facilitation and attenuation of reciprocal la inhibition in ankle extensor and flexor muscles, stabilizing the body.

Studies have shown that jaw opening is associated with head extension (Eriksson et al., 2000) and head position affects the sway of the COG (Makofsky et al., 1991). It is also known that a good balance of both masticatory and head and neck muscles is important for postural stability (Bracco et al., 2004). These reports might explain our finding that in the open jaw position, too, in comparison with the resting jaw position, there is decrease in COG velocity and therefore an increase in body stability.

The body has balance control mechanisms allowing it to maintain its posture as the situation demands. Impaired balance control, either due to aging or to some disease, has been identified as one of the most influential risk factors for falling (Lipsitz and Goldberger, 1992; Goldberger et al., 2002; Close et al., 2005; Stevens et al., 2006; Moreland et al., 2003). Patients with a high risk of falling are characterized by increased variability of postural motion (Morrison et al. 2010), body sway with less overall motion, and increased COG velocity (Hellmann et al. 2011). Consideration of our results may be helpful in the clinical management of patients at higher risk of falling in order to increase their postural stability.

In conclusion, in healthy young adults, changes in voluntary static jaw position can influence the postural stability mechanisms during upright standing both in the presence and in the absence of visual input. Our findings indicate that the jaw sensory-motor system can modulate postural control mechanisms, and might be of value when assessing and planning treatment for patients with postural instability.

\section{Acknowledgment}

This project was financially supported by King Saud University, Vice Deanship of Research Chairs, Rehabilitation Research Chair.

\section{References}

Abrahams VC (1977). The physiology of neck muscles; their role in head movement and maintenance of posture. Can J Physiol Pharmacol 55:332-338.

Blackburn JT, Riemann BL, Myers JB, et al (2003). Kinematic analysis of the hip and trunk during bilateral stance on firm, foam, and multiaxial support surfaces. Clin Biomech (Bristol, Avon) 18:655-661.

Bolmont B, Gangloff P, Vouriot A, et al (2002). Mood states and anxiety influence abilities to maintain balance control in healthy human subjects. Neurosci Lett 329:96-100.

Bracco P, Deregibus A, Piscetta R (2004). Effects of different jaw relations on postural stability in human subjects. Neurosci Lett 356:228-230.
Bracco P, Deregibus A, Piscetta R, et al (1998). Observations on the correlation between posture and jaw position: a pilot study. Cranio 16:252-258.

Brodie AG (1950). Anatomy and physiology of head and neck musculature. Am J Orthod 36:831-844.

Chang CM, Kubota K, Lee MS, et al (1988). Degeneration of the primary snout sensory afferents in the cervical spinal cords following the infraorbital nerve transection in some mammals. Anat Anz 166:43-51.

Cherry EA, Brown LE, Coburn JW, et al (2010). Effect of remote voluntary contractions on knee extensor torque and rate of velocity development. J Strength Cond Res 24:2564-2569.

Chien CW, Hu MH, Tang PF, et al (2007). A comparison of psychometric properties of the smart balance master system and the postural assessment scale for stroke in people who have had mild stroke. Arch Phys Med Rehabil 88:374380.

Clark GT, Browne PA, Nakano M, et al (1993). Co-activation of sternocleidomastoid muscles during maximum clenching. J Dent Res 72:1499-1502.

Close JC, Lord SL, Menz HB, et al (2005). What is the role of falls? Best Pract Res Clin Rheumatol 19:913-935.

Colledge NR, Cantley P, Peaston I, et al (1994). Ageing and balance: the measurement of spontaneous sway by posturography. Gerontology 40:273-278.

Collins, JJ, De Luca, CJ (1995). The effects of visual input on open-loop and closed-loop postural control mechanisms. Exp Brain Res 103:151-163.

Davies PL (1979). Electromyographic study of superficial neck muscles in mandibular function. J Dent Res 58:537-538.

Ebben WP (2006). A brief review of concurrent activation potentiation: theoretical and practical constructs. J Strength Cond Res 20:985-991.

Edwards AS (1946). Body sway and vision. J Exp Psychol 36:526-535.

Ehrlich R, Garlick D, Ninio M (1999). The effect of jaw clenching on the electromyographic activities of 2 neck and 2 trunk muscles. J Orofac Pain 13:115-120.

Eriksson PO, Häggman-Henrikson B, Nordh E, et al (2000). Coordinated mandibular and head-neck movements during rhythmic jaw activities in man. J Dent Res 79:1378-1384.

Eriksson PO, Zafar H, Nordh E (1998). Concomitant mandibular and head-neck movements during jaw opening-closing in man. J Oral Rehabil 25:859-870.

Fransson, PA, Hafström A, Karlberg M, et al (2003). Postural control adaptation during galvanic vestibular and vibratory proprioceptive stimulation. IEEE Trans Biomed Eng 50:1310-1319.

Fransson P, Johansson R, Hafström A, et al (2000). Methods for evaluation of postural control adaptation. Gait Posture 12:14-24.

Fujino S, Takahashi T, Ueno T (2010). Influence of voluntary teeth clenching on the stabilization of postural stance disturbed by electrical stimulation of unilateral lower limb. Gait Posture 31:122-125.

Gangloff P, Louis JP, Perrin PP (2000). Dental occlusion modifies gaze and posture stabilization in human subjects. Neurosci Lett 293:203-206.

Gangloff P, Perrin PP (2002). Unilateral trigeminal anaesthesia modifies postural control in human subjects. Neurosci Lett 330:179-182.

Goldberger AL, Peng CK, Lipsitz LA (2002). What is physiologic complexity and how does it change with aging and disease? Neurobiol Aging 23:23-26.

Hellmann D, Giannakopoulos NN, Blaser R, et al (2011). The effect of various jaw motor tasks on body sway. J Oral Rehabil 38:729-736. 
Hiroshi C (2003). [Relation between teeth clenching and grip force production characteristics]. Kokubyo Gakkai Zasshi 70:82-88.

Horak FB, Hlavacka F (2001). Somatosensory loss increases vestibulospinal sensitivity. J Neurophysiol 86:575-585.

Hosoda M, Masuda T, Isozaki K, et al (2007). Effect of occlusion status on the time required for initiation of recovery in response to external disturbances in the standing position. Clin Biomech (Bristol, Avon) 22:369-373.

Kantor E, Poupard L, Le Bozec S, et al (2001). Does body stability depend on postural chain mobility or stability area Neurosci Lett 308:128-132.

Kavounoudias A, Roll R, Roll JP (1998). The plantar sole is a 'dynamometric map' for human balance control. Neuroreport 9:3247-3252.

Kogler A, Lindfors J, Odkvist LM, et al (2000). Postural stability using different neck positions in normal subjects and patients with neck trauma. Acta Otolaryngol 120:151-155.

Lipsitz LA, Goldberger AL (1992). Loss of 'complexity' and aging. Potential applications of fractals and chaos theory to senescence. JAMA 267:1806-1809.

Liston RA, Brouwer BJ (1996). Reliability and validity of measures obtained from stroke patients using the Balance Master. Arch Phys Med Rehabil 77:425-430.

Makofsky HW, Sexton TR, Diamond DZ, et al (1991). The effect of head posture on muscle contact position using the TScan system of occlusal analysis. Cranio 9:316-321.

Massion J (1994). Postural control system. Curr Opin Neurobiol 4:877-887.

Meyer PF, Oddsson LI, De Luca CJ (2004). The role of plantar cutaneous sensation in unperturbed stance. Exp Brain Res 156:505-512.

Milani RS, De Periere DD, Lapeyre L, et al (2000). Relationship between dental occlusion and posture. Cranio 18:127-134.

Miyahara T, Hagiya N, Ohyama T, et al (1996). Modulation of human soleus $\mathrm{H}$ reflex in association with voluntary clenching of the teeth. J Neurophysiol 76:2033-2041.

Mohapatra S, Kukkar KK, Aruin AS (2014). Support surface related changes in feedforward and feedback control of standing posture. J Electromyogr Kinesiol 24:144-152.

Moreland J, Richardson J, Chan DH, et al (2003). Evidence- based guidelines for the secondary prevention of falls in older adults. Gerontology 49:93-116.

Morrison S, Colberg SR, Mariano M, et al (2010). Balance training reduces falls risk in older individuals with type 2 diabetes. Diabetes Care 33:748-750.

Newstead AH, Hinman MR, Tomberlin JA (2005). Reliability of the Berg Balance Scale and balance master limits of stability tests for individuals with brain injury. J Neurol Phys Ther 29:18-23.

Park SH, Kim HJ, Kim JS, et al (2014). Mastication-induced vertigo and nystagmus. J Neurol 261:480-489.

Perinetti G (2006). Dental occlusion and body posture: no detectable correlation. Gait Posture 24:165-168.

Redfern MS, Yardley L, Bronstein AM (2001). Visual influences on balance. J Anxiety Disord 15:81-94.

Rogers MW, Wardman DL, Lord SR, et al (2001). Passive tactile sensory input improves stability during standing. Exp Brain Res 136:514-522.

Stevens JA, Corso PS, Finkelstein EA, et al (2006). The costs of fatal and non-fatal falls among older adults. Inj Prev 12:290-295.

Takada Y, Miyahara T, Tanaka T, et al (2000). Modulation of $\mathrm{H}$ reflex of pretibial muscles and reciprocal la inhibition of soleus muscle during voluntary teeth clenching in humans. J Neurophysiol 83:2063-2070.

Wada M, Sunaga N, Nagai M (2001). Anxiety affects the postural sway of the antero-posterior axis in college students. Neurosci Lett 302:157-159.

Wardman DL, Day BL, Fitzpatrick RC (2003). Position and velocity responses to galvanic vestibular stimulation in human subjects during standing. J Physiol 547:293-299.

Widmalm SE, Lillie JH, Ash Jr MM (1988). Anatomical and electromyographic studies of the digastric muscle. $\mathrm{J}$ Oral Rehabil 15:3-21.

Zafar H, Nordh E, Eriksson PO (2000). Temporal coordination between mandibular and head-neck movements during jaw opening-closing tasks in man. Arch Oral Biol 45:675-682.

Zafar H, Nordh E, Eriksson PO (2002). Spatiotemporal consistency of human mandibular and head-neck movement trajectories during jaw opening-closing tasks. Exp Brain Res 146:70-76. 